\title{
DESORDEM FISIOLÓGICA E PRODUTIVIDADE DE MANGUEIRA CV. PALMER ASSOCIADA À NUTRIÇÃO DE BORO'
}

\author{
LUIRICK FELIX SILVA BARBOSA², ÍTALO HERBERT LUCENA CAVALCANTE, \\ AUGUSTO MIGUEL NASCIMENTO LIMA ${ }^{4}$
}

RESUMO - O Vale do São Francisco constitui o maior produtor nacional de manga em regime irrigado. Na mangueira, além da produtividade, o boro é o nutriente que mais afeta a qualidade dos frutos, e sua deficiência pode provocar distúrbios fisiológicos. Nesse sentido, um experimento foi desenvolvido com o objetivo de avaliar a associação do boro com a incidência de frutos de manga Palmer acometidos com desordem fisiológica e seu efeito na produtividade de frutos. Os tratamentos consistiram em sete diferentes manejos de adubação: $\mathrm{T} 1$ = Duas fertirrigações com $50 \mathrm{~g}$ planta $^{-1} \mathrm{de}_{3} \mathrm{BO}_{3}$ (adubação do produtor); $\mathrm{T} 2=\mathrm{T} 1+$ cinco pulverizações com $\mathrm{H}_{3} \mathrm{BO}_{3}$ [duas primeiras $(0,3 \%)$ e demais $(0,2 \%)$ ]; $\mathrm{T} 3=\mathrm{T} 1+$ cinco pulverizações com $\mathrm{H}_{3} \mathrm{BO}_{3}$ [duas primeiras $(0,3 \%)$ e demais $(0,4 \%)$ ]; $\mathrm{T} 4=\mathrm{T} 1+$ cinco pulverizações com $\mathrm{H}_{3} \mathrm{BO}_{3}$ [duas primeiras $(0,3 \%)$ e demais $(0,6 \%)]$; $\mathrm{T} 5=\mathrm{T} 1+$ duas pulverizações com $\mathrm{H}_{3} \mathrm{BO}_{3}(0,3 \%)+$ três fertirrigações $\left(10 \mathrm{~g}_{\text {planta }}{ }^{-1}\right.$ de $\left.\mathrm{H}_{3} \mathrm{BO}_{3}\right) ; \mathrm{T} 6=\mathrm{T} 1+$ duas pulverizações com $\mathrm{H}_{3} \mathrm{BO}_{3}(0,3 \%)+$ três fertirrigações $\left(20 \mathrm{~g}\right.$ planta $^{-1}$ de $\left.\mathrm{H}_{3} \mathrm{BO}_{3}\right)$; $\mathrm{T} 7=\mathrm{T} 1+$ duas pulverizações com $\mathrm{H}_{3} \mathrm{BO}_{3}(0,3 \%)+$ três fertirrigações $\left(40 \mathrm{~g}\right.$ planta $\left.{ }^{-1} \mathrm{de}_{3} \mathrm{BO}_{3}\right)$. Os resultados demonstram que há evidências de que a deficiência de boro pode provocar a emissão de frutos acometidos por desordem fisiológica e de que o manejo da adubação boratada é eficiente na redução desse problema e, ainda, influencia a produtividade de manga Palmer, destacando-se que o T2 atingiu produtividade média de 35,62 t ha-1 ${ }^{-1}$ embora mais estudos sejam necessários para a recomendação de um sistema de manejo adequado para a cultura.

Termos para indexação: Mangifera indica L., manga, fertilização.

\section{PHYSIOLOGICAL DESORDERS AND FRUIT YIELD OF MANGO CV. PALMER ASSOCIATED TO BORON NUTRITION: BORON FERTILIZING MANAGEMENT}

\begin{abstract}
The São Francisco Valley is the largest producer of mango under irrigated conditions. In the mango crop, beyond yield, boron is the micronutrient that most affects the fruit quality and this deficiency can cause physiological disorders. Accordingly, an experiment was carried out to evaluate the association of boron with incidence of physiological disorder in mango fruits cv. Palmer and his effect in the fruit yield. The treatments consisted on seven different fertilization managements: $\mathrm{T} 1=$ Two fertirrigations with $50 \mathrm{~g}$ plant ${ }^{-1}$ of $\mathrm{H}_{3} \mathrm{BO}_{3}$ (fertilizing of the grower); $\mathrm{T} 2=\mathrm{T} 1+$ five sprays with $\mathrm{H}_{3} \mathrm{BO}_{3}$ [first two $(0.3 \%)$ and others $(0.2 \%)] ; \mathrm{T} 3=\mathrm{T} 1+$ five sprays with $\mathrm{H}_{3} \mathrm{BO}_{3}$ [first two $(0.3 \%)$ and others $\left.(0.4 \%)\right] ; \mathrm{T} 4=\mathrm{T} 1+$ five sprays with $\mathrm{H}_{3} \mathrm{BO}_{3}$ [first two $(0.3 \%)$ and others $\left.(0.6 \%)\right] ; \mathrm{T} 5=\mathrm{T} 1+$ two sprays with $\mathrm{H}_{3} \mathrm{BO}_{3}(0.3 \%)+$ three fertirrigations $\left(10 \mathrm{~g} \mathrm{plant}^{-1}\right.$ of $\left.\mathrm{H}_{3} \mathrm{BO}_{3}\right)$; $\mathrm{T} 6=\mathrm{T} 1+$ two sprays with $\mathrm{H}_{3} \mathrm{BO}_{3}(0.3 \%)+$ three fertirrigations $\left(20 \mathrm{~g}\right.$ plant $^{-1}$ of $\left.\mathrm{H}_{3} \mathrm{BO}_{3}\right) ; \mathrm{T} 7=\mathrm{T} 1+$ two sprays with $\mathrm{H}_{3} \mathrm{BO}_{3}(0.3 \%)+$ three fertirrigations $\left(40 \mathrm{~g} \mathrm{plant}^{-1}\right.$ of $\left._{3} \mathrm{BO}_{3}\right)$. There is evidence that boron deficiency can cause the emission of fruits affected by physiological disorder and the management of boron fertilization is effective for reducing this problem and also influences mango fruit yield cv. Palmer, detaching that T2 reached an average yield of $35.62 \mathrm{t} \mathrm{ha}^{-1}$, although more studies are needed to recommend a suitable crop management system.
\end{abstract}

Index terms: Mangifera indica L., mango, fertilizing.

\footnotetext{
'(Trabalho 273-14). Recebido em: 28-10-2014. Aceito para publicação em: 04-08-2015.

${ }^{2}$ Graduando em Engenharia Agronômica, Universidade Federal do Vale do São Francisco. Rodovia BR 407 - KM 12 - Lote 543 PSNC, s/n ${ }^{\circ}$ - C1, CEP: 56300-990, Petrolina, PE. Bolsista PIBIC/CNPq. E-mail: luirick.barbosa@gmail.com

${ }^{3}$ Dr., Professor, Universidade Federal do Vale do São Francisco, Petrolina, PE. Bolsista produtividade em Pesquisa do CNPq. E-mail:italo.cavalcante@univasf.edu.br*Autor para correspondência

${ }^{4}$ Dr., Professor, Universidade Federal do Vale do São Francisco, Petrolina-PE. E-mail: augusto.lima@univasf.edu.br
} 


\section{INTRODUÇÃO}

O Vale do São Francisco constitui o maior produtor nacional de manga em regime irrigado, produzindo aproximadamente $250.000 \mathrm{t} \mathrm{ano}^{-1} \mathrm{e}$ respondendo por mais de $90 \%$ das exportações brasileiras dessa fruta in natura, o que corresponde a quase $125.000 \mathrm{t}$ (IBRAF, 2014) e contribui para que o Brasil seja o quarto maior exportador mundial de manga com quase $10 \%$ do mercado (FAO, 2014).

Dentre as cultivares de manga mais produzidas no Vale do São Francisco, destacam-se Tommy Atkins, Palmer, Kent, Keitt, entre outras. A 'Palmer' tem-se caracterizado como a cultivar de maior aceitação no mercado interno devido ao sabor e à produção semitardia, com frutos de polpa amarela firme com pouca ou nenhuma fibra e casca fina, bom sabor, relação polpa/fruto de $72 \%$ e pouco suscetível ao colapso interno no fruto (GENÚ; PINTO, 2002). Contudo, essa variedade tem apresentado, no Vale do São Francisco, a incidência de frutos pequenos, sem sementes e com formato diferente, vulgarmente conhecido como "manguita" ou "castanha", o que pode estar associado à nutrição boratada.

Na mangueira, além da produtividade, o boro é o que mais afeta a qualidade dos frutos (GALLI, 2012), pois quando em baixos níveis resulta em menor florescimento e polinização, além de frutos de tamanho reduzido (SILVA et al., 2014). A floração dessa planta é geralmente excessiva, no entanto em algumas situações há baixo pegamento dos frutos e queda prematura, o que pode estar relacionado com a importância do boro na germinação do grão de pólen e no crescimento do tubo polínico (SARAN; KUMAR, 2011).

A deficiência de boro pode provocar vários distúrbios fisiológicos, dentre os quais, a necrose interna, que foi identificada como o mais novo distúrbio em frutos de manga em desenvolvimento, mas também há relatos da ocorrência de sementes necrosadas, frutos com a ponta mais fina e frutos rachados (SARAN; KUMAR, 2011).

Como fonte de boro, o ácido bórico é o mais utilizado nas adubações (LEMISKA et al., 2014), sendo que quando aplicado no solo supre os frutos e, quando aplicado via foliar, aumenta os teores de boro nas flores, sendo que ambas as aplicações refletem em melhora na produtividade (WÓJCIK et al., 2008). Saran e Kumar (2011) concluíram que uma aplicação equilibrada de macronutrientes, juntamente com o manejo adequado da adubação boratada, é imperativa para a produção de qualidade de manga.

Nesse sentido, este trabalho foi desenvolvido com o objetivo de avaliar a associação do boro com a incidência de frutos de manga Palmer acometidos com desordem fisiológica vulgarmente conhecida como "manguita"; e o manejo de adubação boratada visando ao combate a essa desordem e ao aumento da produtividade da mangueira Palmer cultivada no Vale do São Francisco.

\section{MATERIAL E MÉTODOS}

O estudo foi realizado em parceria com a fazenda Herculano Agrícola localizada no município de Casa Nova - BA, com coordenadas geográficas $09^{\circ} 11^{\prime} 43,5$ " latitude sul, $41^{\circ} 01^{\prime} 59,2$ " longitude oeste e altitude de 400,3 m. Estudaram-se plantas da variedade Palmer, com 11 anos de idade, plantadas em espaçamento $7 \times 7 \mathrm{~m}$, irrigadas pelo sistema localizado de microaspersão. Segundo a classificação de Köppen, o clima local é do tipo Bswh' (semiárido), com precipitação inferior a $500 \mathrm{~mm}$ concentrada em três a quatro meses do ano. Durante o experimento, a maior temperatura e a umidade relativa do ar observada foram de $35,36^{\circ} \mathrm{C}$ e $88,70 \%$, enquanto os menores valores registrados foram de $20,68^{\circ} \mathrm{C}$ e $22,33 \%$, tendo como média, durante todo o trabalho, $26,66^{\circ} \mathrm{C}$ e $61,44 \%$, respectivamente.

Antes da instalação do experimento, foi coletada amostra de solo na camada de $0-40 \mathrm{~cm}$ de profundidade, retirando-se 20 amostras simples para obtenção de amostra composta. A amostra de solo foi seca ao ar, destorroada, homogeneizada e passada em peneira de malha de 2,0 $\mathrm{mm}$ para a obtenção da terra fina seca ao ar (TFSA). Para caracterização química, foram determinados os valores de condutividade elétrica no extrato da pasta de saturação $\left(\mathrm{CE}_{\mathrm{es}}\right), \mathrm{pH}$ $\left(\mathrm{H}_{2} \mathrm{O}\right)$, acidez potencial $(\mathrm{H}+\mathrm{Al})$, acidez trocável $\left(\mathrm{Al}^{3+}\right)$, teor de matéria orgânica do solo, conforme metodologia proposta por Silva (2009) (Tabela 1). Foram determinados, também, os teores de fósforo $(\mathrm{P})$, cálcio $\left(\mathrm{Ca}^{2+}\right)$, magnésio $\left(\mathrm{Mg}^{2+}\right)$, potássio $\left(\mathrm{K}^{+}\right) \mathrm{e}$ sódio $\left(\mathrm{Na}^{+}\right)$, e calculadas a CTC e a saturação por bases (V), segundo metodologia proposta por Silva (2009). Além disso, foram determinados também os teores de boro $(\mathrm{B})$, ferro $(\mathrm{Fe})$, manganês $(\mathrm{Mn})$, cobre $(\mathrm{Cu})$ e zinco $(\mathrm{Zn})$, sendo a leitura realizada no espectrofotômetro de absorção atômica (EAA).

$\mathrm{O}$ delineamento experimental empregado foi o inteiramente casualizado, com sete tratamentos e quatro repetições de duas plantas cada, mantendose duas plantas bordaduras entre as parcelas e no perímetro experimental. Os tratamentos consistiram em:

$\mathrm{T} 1=$ Duas fertirrigações com $50 \mathrm{~g}_{\text {planta }}{ }^{-1} \mathrm{de}$ $\mathrm{H}_{3} \mathrm{BO}_{3}$ (adubação do produtor);

$\mathrm{T} 2=\mathrm{T} 1+$ cinco pulverizações com $\mathrm{H}_{3} \mathrm{BO}_{3}$ 
[duas primeiras $(0,3 \%)$ e demais $(0,2 \%)]$;

$\mathrm{T} 3=\mathrm{T} 1+$ cinco pulverizações com $\mathrm{H}_{3} \mathrm{BO}_{3}$ [duas primeiras $(0,3 \%)$ e demais $(0,4 \%)$ ];

$\mathrm{T} 4=\mathrm{T} 1+$ cinco pulverizações com $\mathrm{H}_{3} \mathrm{BO}_{3}$ [duas primeiras $(0,3 \%)$ e demais $(0,6 \%)$ ];

$\mathrm{T} 5=\mathrm{T} 1+$ duas pulverizações com $\mathrm{H}_{3} \mathrm{BO}_{3}$ $(0,3 \%)+$ três fertirrigações $\left(10\right.$ g planta $\left.^{-1} \mathrm{de}_{3} \mathrm{BO}_{3}\right)$;

$\mathrm{T} 6=\mathrm{T} 1+$ duas pulverizações com $\mathrm{H}_{3} \mathrm{BO}_{3}$ $(0,3 \%)+$ três fertirrigações $\left(20\right.$ g planta $\left.^{-1} \mathrm{de}_{3} \mathrm{BO}_{3}\right)$;

$\mathrm{T} 7=\mathrm{T} 1+$ duas pulverizações com $\mathrm{H}_{3} \mathrm{BO}_{3}$ $(0,3 \%)+$ três fertirrigações $\left(40 \mathrm{~g} \mathrm{planta}^{-1} \mathrm{de}_{3} \mathrm{BO}_{3}\right)$.

A adubação boratada comumente utilizada pelos produtores da região é realizada em duas fertirrigações com $50 \mathrm{~g} \mathrm{planta}^{-1}$ de $_{3} \mathrm{BO}_{3}$ com a planta em pleno florescimento. Todas as adubações dos tratamentos T2 a T7 foram realizadas em dezembro de 2013, iniciando quando as panículas se encontravam recentemente emitidas (aproximadamente aos sete dias após o início da emissão). A definição dos tratamentos foi feita compilando as recomendações da literatura para adubação foliar e fertirrigação (GENÚ; PINTO, 2002), considerando a demanda da cultura.

As plantas foram submetidas às práticas culturais recomendadas para a cultura da mangueira nas condições regionais de cultivo, seguindo as recomendações de Genú e Pinto et al. (2002). Visando ao estimulo de novas brotações a partir das gemas axilares, foi realizada a poda, onde foram feitos cortes dos ramos sempre acima do internódio (ou nó). Quando os novos brotos se encontravam no segundo fluxo vegetativo, foi aplicado na projeção da copa o Paclobutrazol (PBZ) para inibir a biossíntese das giberelinas e, com isso, ocasionar a paralisação do alongamento das brotações, o crescimento vegetativo $\mathrm{e}$, consequentemente, promover o amadurecimento dos ramos. Um mês após o travamento, teve-se o início da indução floral com sete aplicações de nitrato de cálcio, espaçadas a cada cinco dias, e com concentrações variando entre 2 a $4 \%$. Paralelamente ao travamento e à indução floral, foi suspensa aplicação de água com o intuito de promover estresse hídrico na planta, só retornando a aplicação com o início do florescimento.

Após a ocorrência da queda fisiológica, os frutos que apresentaram desordem fisiológica, vulgarmente conhecida como "manguita" ou "castanha", foram retirados das plantas e contados para a determinação do número de frutos com desordem por planta. A desordem fisiológica é caracterizada por apresentar frutos pequenos, sem sementes e com formato diferente, conforme se pode observar na Figura 1.

Após o florescimento, foram coletadas amostras de folhas em todos os quadrantes da planta, a uma altura mediana da copa, em ramos com flores e retirando as folhas da parte mediana do ramo para determinar o teor de boro nas plantas, seguindo as recomendações de Silva (2009).

Para fins de determinação de produtividade, foram colhidos apenas os frutos com tamanho mínimo de $15 \mathrm{~cm}$ e maturidade fisiológica, definida a partir da coloração da polpa (creme amarela), escala de coloração indicada pelo Programa Brasileiro para a Modernização da Horticultura (2004). A colheita foi realizada manualmente e no período da manhã.

Os dados foram submetidos à análise de variância para o diagnóstico do efeito significativo entre as médias dos tratamentos em estudo e a correlação e regressão entre os teores foliares de boro e o número de "manguitas" por planta. A análise de variância e a regressão foram feitas no programa estatístico ASSISTAT, enquanto a regressão e a confecção dos gráficos, no Sigma Plot 10.0 .

\section{RESULTADOS E DISCUSSÃO}

A média dos resultados obtidos da quantificação de frutos não comerciais, com desordem fisiológica "manguita", encontra-se na Figura 2, onde se verifica que os diferentes manejos de fertilização boratada apresentaram diferença significativa em relação ao tratamento $\mathrm{T} 1$ (adubação do produtor). O tratamento T1 (adubação do produtor) apresentou, em média, 239 mangas, planta $^{-1}$ com desordem fisiológica, enquanto para os demais tratamentos a média de mangas $/$ planta $^{-1}$ com desordem fisiológica foi aproximadamente sete. Saran e Kumar (2011) aplicaram boro via solo e foliar com o objetivo de reduzir a incidência de frutos rachados, na Índia, e obtiveram, quando aplicados $25 ; 50$ e 75 g planta $^{-1}$ via solo, redução de $2,02 \%, 1,01 \%$ e $1,05 \%$, respectivamente, enquanto via foliar, $0,05 \%, 0,075 \%$ e $0,10 \%$ de boro, reduziu $0,90 \%, 0,75 \%$ e $0,47 \%$, respectivamente, o número de frutos rachados.

Moazzam et al. (2011) avaliaram o efeito da aplicação foliar de diferentes combinações de ferro ( $\mathrm{Fe}$ ), boro (B) e zinco ( $\mathrm{Zn})$ sobre a qualidade dos frutos da mangueira cv. 'Dashehari', na Índia, e constataram que a aplicação desses micronutrientes aumentou expressivamente a qualidade dos frutos. Para Bhattet al. (2012), o boro, juntamente com zinco desempenham funções importantes na formação e na manutenção da qualidade dos frutos da mangueira. Em fruteiras, a deficiência boratada resulta em mau funcionamento do tecido do câmbio vascular, 
responsável pela multiplicação de células dos vasos condutores, culminando em colapso imediato do floema e do xilema, em deficiência aguda. Com isso, há um menor transporte de fotoassimilados para o sistema radicular, reduzindo seu crescimento, prejudicando a absorção de água e de nutrientes. Como ocorre redução no transporte de açúcares para as raízes, os frutos podem acumular essas substâncias em bolsas de goma no albedo, afetando sua qualidade (QUAGGIO et al., 2003).

O boro é um micronutriente essencial para as plantas, participando diretamente da polinização e do desenvolvimento de frutos e essencial para a absorção e uso de cálcio, apresentando importante papel na resistência das paredes celulares (GENÚ; PINTO, 2002). Rajput et al. (1976), pulverizando mangueiras com ácido bórico a $0,8 \%$ antes do florescimento, na Índia, obtiveram aumento de 95\% na produção de frutos por panícula. A deficiência de boro ocasiona empobrecimento no florescimento e na polinização além de frutos de tamanhos reduzidos, sendo mais comum em solos arenosos (BOLOGNA; VITTI, 2006), como no presente trabalho. Além disso, a carência de boro parece acelerar a produção da enzima polifenolase e favorecer o maior desenvolvimento de fenólicos no embrião, comprometendo a qualidade dos frutos (GENÚ; PINTO, 2002). Avaliando o efeito da fertilização boratada no rendimento e na qualidade de frutos de macieira cv. Imperial Gala e Fuji Suprema, em São Joaquim - SC, Sá et al. (2014) observaram que a fertilização de B foliar aumentou o índice de iodo-amido, que passou de 3,0, quando não houve aplicação foliar de B, para 4,1, quando a pulverização foliar ocorreu na floração. Assim, fertilização foliar de $\mathrm{B}$ resultou em maior degradação do amido em açúcares solúveis, antecipando a maturação dos frutos. A fertilização foliar de B diminuiu em 19\% a acidez titulável em relação à ausência da fertilização foliar de B na 'Imperial Gala', e em 14\% na 'Fuji Suprema', mostrando também uma antecipação da maturação dos frutos.

Na Figura 3, é possível observar, no tratamento T1, plantas com frutos pequenos e a quantidade de frutos não comerciais retirados de uma mesma planta. A fertilização com boro para atender à demanda das plantas pode ser realizada através de aplicações de fertilizantes diretamente no solo ou nas folhas. No entanto, um dos principais problemas práticos da aplicação via solo está relacionado à dificuldade de distribuição uniforme do nutriente no pomar, em virtude da pequena quantidade recomendada, além do potencial de perdas por lixiviação. A elevada solubilidade da maioria dos fertilizantes boratados resulta em maior mobilidade no solo, com consequente lixiviação, principalmente em solos arenosos. No solo, a concentração de B na solução e sua disponibilidade são fortemente afetadas por reações de adsorção química aos grupos funcionais existentes na superfície de compostos orgânicos e inorgânicos e pelo estoque de matéria orgânica (GOLDBERG, 1993). Por outro lado, a fertilização foliar é benéfica no momento em que as raízes têm dificuldades em absorver rapidamente o boro necessário para atender à demanda da planta. Adicionalmente, o custo da fertilização foliar com micronutrientes, normalmente, não é oneroso comparado ao valor da cultura e com a aplicação no solo, podendo ser aplicados juntamente com os produtos fitossanitários, pois são utilizadas quantidades muito pequenas.

Os teores foliares de B apresentaram correlações significativas e negativas com o número de frutos com desordem fisiológica "manguita", ou seja, à medida que aumenta a concentração de boro nas folhas, ocorre uma redução no número de "manguitas" por planta (Figura 4). Saran e Kumar (2011) constataram aumento nas concentrações de boro em folhas de mangueira "Dashehari" com o incremento da dose aplicada, com maior eficiência quando aplicado via foliar. Adicionalmente, esses autores, ainda, relataram que o manejo da adubação boratada reduziu a incidência de frutos rachados.

Para o desenvolvimento adequado das culturas, o teor de boro na planta é bastante variável, diferindo quanto à exigência devido à composição química das paredes celulares das diversas espécies (BASTOS; CARVALHO, 2004). Para Genú e Pinto (2002), a faixa adequada para o teor de boro foliar em mangueiras varia de 50 a $100 \mathrm{mg} \mathrm{kg}^{-1}$. Para a variedade Palmer do presente estudo, a faixa de suficiência estabelecida pela literatura não atende à demanda nutricional da cultura para esse micronutriente, favorecendo o desenvolvimento de frutos com desordem fisiológica "manguita". O tratamento T1 (adubação do produtor), com maior produção de frutos com desordem fisiológica "manguita", apresentou $120 \mathrm{mg} \mathrm{kg}^{-1}$ de boro foliar, estando acima da faixa de suficiência estabelecida pela literatura. Apesar das valiosas informações contidas nos valores de referências da literatura para a interpretação dos resultados de análises foliares, o sucesso na interpretação dessas análises está condicionado à obtenção de padrões de referências "Normas" que podem ser obtidos em condições específicas de clima, solo e manejo do pomar. Por outro lado, não se verificaram sintomas visuais de deficiência de $\mathrm{B}$ em quaisquer dos tratamentos 
estudados.

A produtividade $\left(\mathrm{t} \mathrm{ha}^{-1}\right)$ de manga Palmer foi significativamente influenciada pelo manejo da adubação boratada (Figura 5). Observa-se que a adubação boratada para a mangueira Palmer não foi suficientemente eficiente quando aplicada apenas via fertirrigação, pois o tratamento T1 (adubação do produtor) apresentou média significativamente inferior aos demais tratamentos, permitindo constatar a importância da adubação boratada foliar para a produtividade da mangueira Palmer, especialmente porque esse tratamento (T1) também apresentou o maior número de frutos com incidência de desordem fisiológica associada ao boro (Figura 2). Nesse sentido, Bhatt et al. (2012), em estudo desenvolvido na Índia com o objetivo de proporcionar uma resposta rápida, via pulverização foliar, para a correção de deficiências de micronutrientes, dentre os quais o boro, concluíram que a aplicação foliar de $0,5 \%$ de bórax incrementou a produtividade da manga Dashehari.

A adubação do produtor (T1) apresentou a menor média de produtividade dentre os tratamentos avaliados $\left(15,6 \mathrm{tha}^{-1}\right)$, inclusive inferior com a média do Vale do Submédio São Francisco $\left(20 \mathrm{t} \mathrm{ha}^{-1}\right)$, mas, ainda, compatível à média nacional $\left(16 \mathrm{tha}^{-1}\right)$ (IBGE, 2012). Apesar de o teor de B no solo do presente trabalho $\left(0,40 \mathrm{mg} \mathrm{dm}^{-3}\right)$ (Tabela 1) ser considerado médio (0,36-0,60 $\left.\mathrm{mg} \mathrm{dm}^{-3}\right)$ (Alvarez et al., 1999), este está abaixo do nível crítico $\left(0,60 \mathrm{mg} \mathrm{dm}^{-3}\right)$, apresentando potencial de resposta à adubação. $\mathrm{O}$ nível crítico é definido como o teor de nutriente no solo correspondente à disponibilidade para obter a produção de máxima eficiência econômica, quando os demais nutrientes e fatores de produção estão próximo ao nível adequado (Alvarez et al., 1999). Além disso, a maioria das tabelas de interpretação de disponibilidade de macro e micronutrientes foi desenvolvida sob condição de cultivo não irrigado, mostrando-se a necessidade de estudos voltados para o estabelecimento de classes de disponibilidade de $B$ em condições sob irrigação. Adicionalmente, cabe ressaltar, ainda, que o manejo adequado da adubação boratada no sistema solo-planta é normalmente dificultado, em virtude de a faixa de concentração entre a deficiência e a toxicidade ser estreita, se comparada à dos outros nutrientes (PRADO et al. 2006).

Entre a adubação do produtor (T1) e o tratamento T2 $\left[\mathrm{T} 1+\right.$ cinco pulverizações com $\mathrm{H}_{3} \mathrm{BO}_{3}$ (duas primeiras de $0,3 \%$ e demais $0,2 \%$ )], houve incremento de produtividade de aproximadamente $133 \%$, o que pode ser atribuído às funções que o boro exerce na planta quanto a fatores diretamente relacionados ao florescimento e à produção de frutos, conforme destaca Sá et al. (2014). Assim, o presente trabalho mostra claramente os benefícios da adubação foliar adicionalmente à adubação via solo (fertirrigação) para o boro. Apesar da limitada mobilidade do B na planta, a adubação foliar normalmente possibilita alto índice de utilização do nutriente pela planta e respostas rápidas, sendo possível, inclusive, corrigir deficiências após o seu aparecimento. Assim, a adubação foliar em complemento à fertirrigação foi extremamente importante para reduzir a desordem fisiológica "manguita" e elevar a produtividade da cultura da mangueira.

O B tem sido historicamente considerado um nutriente de baixa mobilidade no floema, em virtude de concentrações mais elevadas serem encontradas nas partes mais velhas das plantas, e os sintomas de deficiências inicialmente aparecerem nas partes mais novas (BASTOS; CARVALHO, 2004; RAVEN, 1980). No entanto, Brown e Shelp (1997) informam que o B pode ser redistribuído em algumas espécies de plantas, que utilizam polióis como metabólitos secundários, culminando na formação de complexo poliol-B-poliol nos tecidos vegetais fotossintéticos. Assim, segundo Brown e Shelp (1997) e Perica et al. (2001) o B pode ser transportado no floema, para diversos drenos ativos, incluindo folhas jovens, frutos, sementes e meristemas vegetativos ou reprodutivos. Com isso, a fertilização foliar boratada possibilita uma rápida absorção e translocação do B para outras partes da planta, constituindo uma excelente estratégia de manejo quando se busca uma rápida resposta à fertilização boratada.

A produtividade média de $35,62 \mathrm{t} \mathrm{ha}^{-1}$ registrada no T2 está muito acima da média da região do Vale do Submédio São Francisco $\left(20 \mathrm{tha}^{-1}\right)$, superior às $10,5 \mathrm{t}$ $\mathrm{ha}^{-1}$ de manga Palmer reportada por Pleguezuelo et al. (2012) na Espanha; e aos 6,4 t ha $\mathrm{a}^{-1}$ de manga Palmer observada por Silva et al. (2014) em São Paulo. Vale destacar que, na determinação de produtividade do presente trabalho, apenas se consideraram frutos de qualidade, i.e., com comprimento acima de $15 \mathrm{~cm}$ e maturidade fisiológica definida a partir da coloração da polpa (creme amarela), diferentemente das estatísticas oficiais ou do registrado por Pleguezuelo et al. (2012) e Silva et al. (2014), os quais consideram produtividade total. 


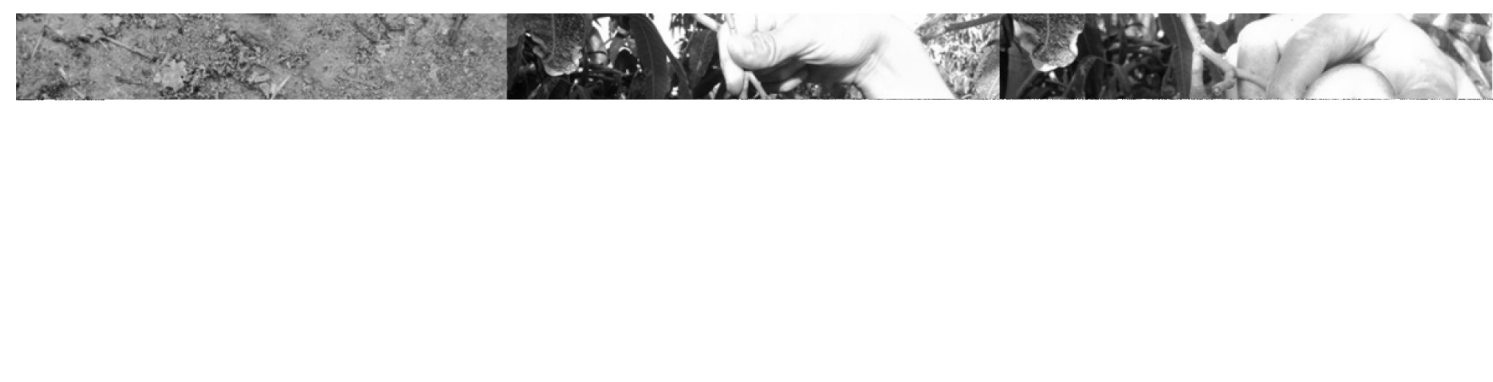

FIGURA 1 -Frutos de manga Palmer caracterizados como "manguita" ou "castanha".

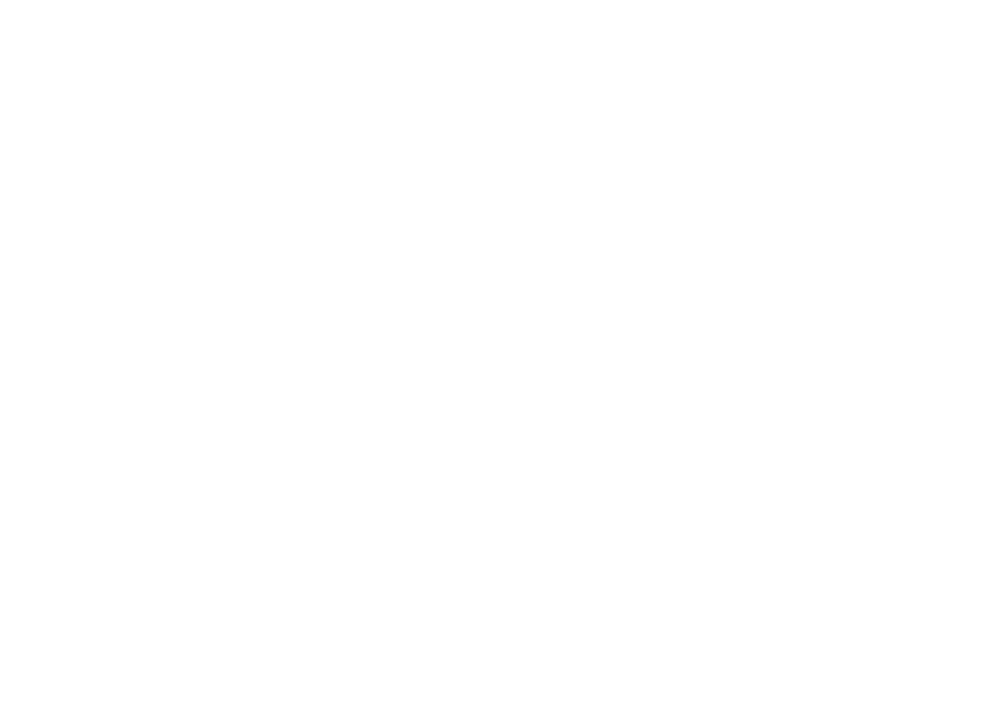

FIGURA 2 - Número médio de frutos que apresentaram desordem fisiológica em função do manejo de adubação boratada na mangueira Palmer, com respectivas barras de erro-padrão $(n=8)$. Barras com mesma letra não diferem entre si, pelo teste de Tukey $(p<0,01)$. T1 $=$ Duas fertirrigações

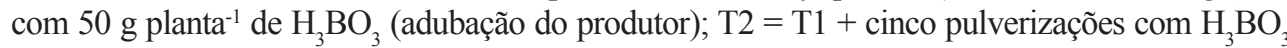
[duas primeiras $(0,3 \%)$ e demais $(0,2 \%)$ ]; $\mathrm{T} 3=\mathrm{T} 1+$ cinco pulverizações com $\mathrm{H}_{3} \mathrm{BO}_{3}$ [duas primeiras $(0,3 \%)$ e demais $(0,4 \%)$; $\mathrm{T} 4=\mathrm{T} 1+$ cinco pulverizações com $\mathrm{H}_{3} \mathrm{BO}_{3}$ [duas primeiras $(0,3 \%)$ e demais $(0,6 \%)] ; \mathrm{T} 5=\mathrm{T} 1+$ duas pulverizações com $\mathrm{H}_{3} \mathrm{BO}_{3}(0,3 \%)+$ três fertirrigações $\left(10\right.$ g planta $^{-1}$ de $\left._{3} \mathrm{BO}_{3}\right) ; \mathrm{T} 6=\mathrm{T} 1+$ duas pulverizações com $\mathrm{H}_{3} \mathrm{BO}_{3}(0,3 \%)+$ três fertirrigações $(20$ g planta $^{-1}$ de $\left.\mathrm{H}_{3} \mathrm{BO}_{3}\right) ; \mathrm{T} 7=\mathrm{T} 1+$ duas pulverizações com $\mathrm{H}_{3} \mathrm{BO}_{3}(0,3 \%)+$ três fertirrigações $(40 \mathrm{~g}$ planta $^{-1}$ de $\mathrm{H}_{3} \mathrm{BO}_{3}$ ).

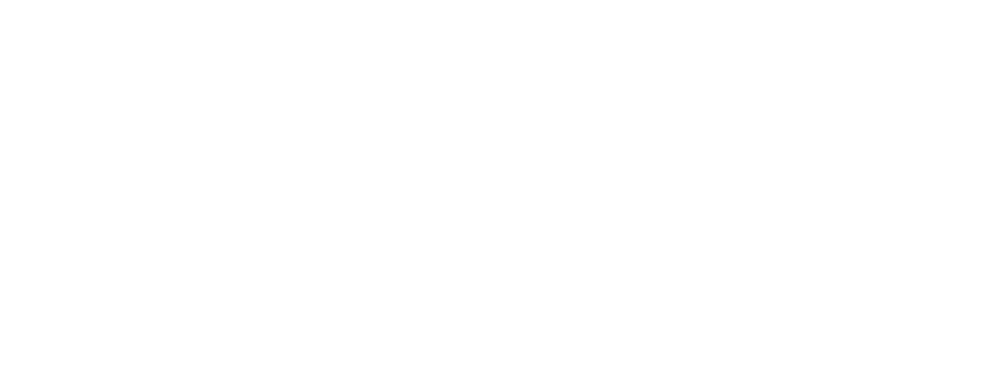

FIGURA 3 - Frutos de manga Palmer caracterizados como "manguita" ou "castanha"(A) e "manguitas" colhidas em uma das plantas do tratamento T1 (B). 


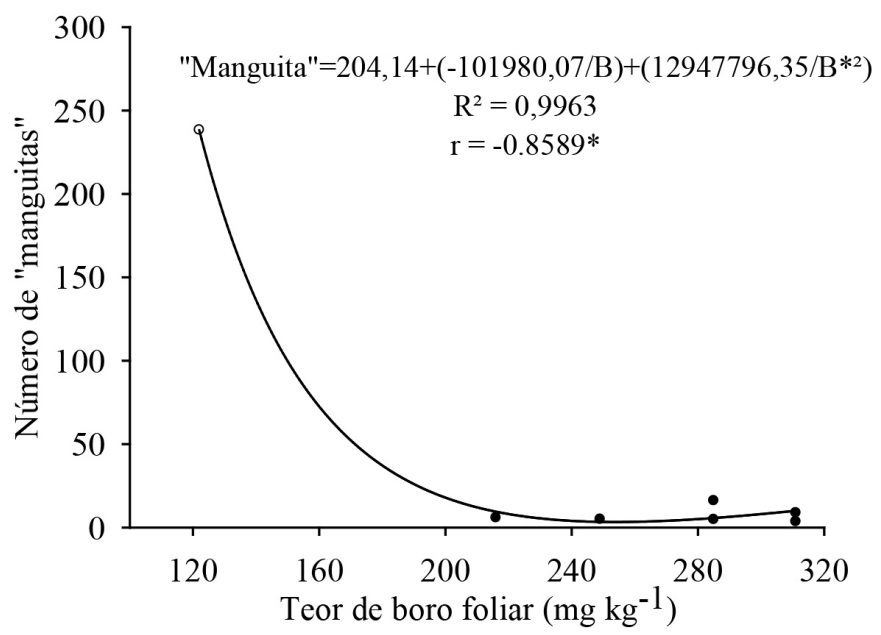

FIGURA 4 - Correlação entre o teor de boro foliar e número de "manguitas" em mangueira Palmer. Significativo para $(p<0,05)$.

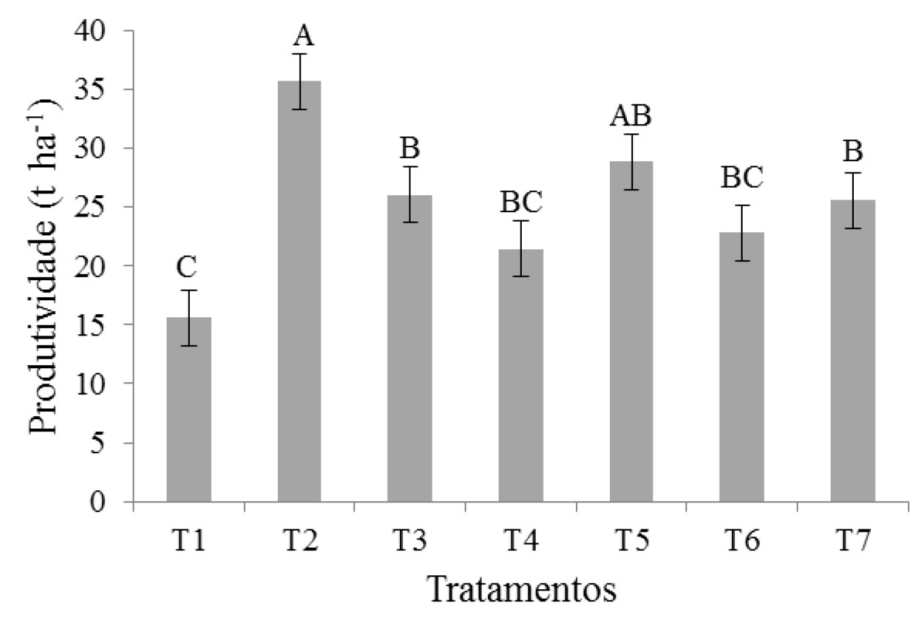

FIGURA 5 - Produtividade $\left(\mathrm{t} \mathrm{ha}^{-1}\right)$ de manga Palmer em função do manejo de adubação boratada com respectivas barras de erro-padrão $(\mathrm{n}=8)$. Barras com mesma letra não diferem entre si, pelo teste de Tukey $(p<$ 0,01). $\mathrm{T} 1=$ Duas fertirrigações com $50 \mathrm{~g}_{\text {planta }}{ }^{-1} \mathrm{de}_{3} \mathrm{BO}_{3}$ (adubação do produtor); $\mathrm{T} 2=\mathrm{T} 1+$ cinco pulverizações com $\mathrm{H}_{3} \mathrm{BO}_{3}$ [duas primeiras $(0,3 \%)$ e demais $(0,2 \%)$ ]; $\mathrm{T} 3=\mathrm{T} 1+$ cinco pulverizações com $\mathrm{H}_{3} \mathrm{BO}_{3}$ [duas primeiras $(0,3 \%)$ e demais $(0,4 \%)$ ]; $\mathrm{T} 4=\mathrm{T} 1+$ cinco pulverizações com $\mathrm{H}_{3} \mathrm{BO}_{3}$ [duas primeiras $(0,3 \%)$ e demais $(0,6 \%)$ ]; $\mathrm{T} 5=\mathrm{T} 1+$ duas pulverizações com $\mathrm{H}_{3} \mathrm{BO}_{3}(0,3 \%)+$ três fertirrigações $\left(10\right.$ g planta $^{-1}$ de $\left.\mathrm{H}_{3} \mathrm{BO}_{3}\right)$; $\mathrm{T} 6=\mathrm{T} 1+$ duas pulverizações com $\mathrm{H}_{3} \mathrm{BO}_{3}(0,3 \%)+$ três fertirrigações $\left(20\right.$ g planta $^{-1}$ de $\left.\mathrm{H}_{3} \mathrm{BO}_{3}\right) ; \mathrm{T} 7=\mathrm{T} 1+$ duas pulverizações com $\mathrm{H}_{3} \mathrm{BO}_{3}(0,3 \%)+$ três fertirrigações ( $40 \mathrm{~g} \mathrm{planta}^{-1}$ de $\mathrm{H}_{3} \mathrm{BO}_{3}$ ). 
TABELA 1 - Características químicas do solo sob cultivo de mangueira Palmer antes da instalação do experimento.

\begin{tabular}{|c|c|c|c|c|c|c|c|c|}
\hline CEes & MO & $\mathrm{pH}\left(\mathrm{H}_{2} \mathrm{O}\right)$ & $\mathbf{P}$ & $\mathbf{K}^{+}$ & $\mathrm{Ca}^{2+}$ & $\mathrm{Mg}^{2+}$ & \multicolumn{2}{|c|}{$\mathrm{Na}^{+}$} \\
\hline $\mathrm{dS} \mathrm{m}^{-1}$ & $\mathrm{~g} \mathrm{~kg}^{-1}$ & & $\mathrm{mg} \mathrm{dm}^{-3}$ & & & $\mathrm{cmol}_{\mathrm{c}} \mathrm{dm}^{-3}$ & & \\
\hline 0,30 & 19,00 & 6,5 & 36,00 & 0,32 & 4,3 & 1,2 & \multicolumn{2}{|c|}{0,05} \\
\hline $\mathrm{H}+\mathrm{Al}$ & CTC & $\mathrm{Al}^{3+}$ & $\mathbf{V}$ & $\mathrm{Cu}$ & $\mathbf{F e}$ & Mn & $\mathbf{Z n}$ & B \\
\hline 0,96 & $\begin{array}{c}\mathrm{cmol}_{\mathrm{c}} \mathrm{dm}^{-3}-8,83 \\
\end{array}$ & $\overline{0,00}$ & $\begin{array}{c}-\%- \\
86,00\end{array}$ & 0,60 & 3,10 & $\begin{array}{c}-\mathrm{mg} \mathrm{dm}^{-3}- \\
33,00\end{array}$ & 49,80 & 0,40 \\
\hline
\end{tabular}

CEes: Condutividade elétrica no extrato de saturação; MO: Matéria orgânica; $\mathrm{H}+\mathrm{Al}$ : Acidez potencial; CTC: Capacidade de troca de cátions; V: Saturação por bases.

\section{CONCLUSÃO}

Há evidências de que a deficiência de boro pode provocar a emissão de frutos acometidos por desordem fisiológica, conhecida vulgarmente como "manguita", e o seu manejo de adubação pode ser eficiente na redução desse problema.

O manejo da adubação boratada influencia na produtividade de manga Palmer com destaque para o manejo que inclui duas fertirrigações com 50 g planta ${ }^{-1}$ de $\mathrm{H}_{3} \mathrm{BO}_{3}+$ cinco pulverizações com $\mathrm{H}_{3} \mathrm{BO}_{3}$ [duas primeiras $(0,3 \%)$ e demais $(0,2 \%)$ ] que atingiu produtividade média de 35,62 $\mathrm{t} \mathrm{ha}^{-1}$, embora mais estudos sejam necessários para recomendação de um sistema de manejo adequado para a cultura.

\section{REFERÊNCIAS}

ALVAREZ V., V.H.; NOVAIS, R.F.; BARROS, N.F.; CANTARUTTI, R.B.; LOPES, A.L. Interpretação dos resultados das analises de solo. In: RIBEIRO, A.C.; GUIMARÃES, P.T.G.; ALVAREZ V.H. (Ed.). Recomendações para o uso de corretivos e fertilizantes em Minas Gerais: $5^{a}$ Aproximação. Viçosa: CFSEMG, 1999. p.25-32.

BASTOS, A.R.R.; CARVALHO, J.G. de. Absorção radicular e redistribuição do boro pelas plantas, e seu papel na parede celular. Revista Universidade Rural: Série Ciências da Vida, Rio de Janeiro, v.24, n.2, p.47-66, 2004.

BHATT, A.; MISHRA, N.; MISHRA, D.; SINGH, C. Foliar application of potassium, calcium, zinc and boron enhanced yield, quality and shelf life of mango. HortFlora Research Spectrum, New Delhi, v.1, n.4, p.300-305, 2012.
BOLOGNA, I.R.; VITTI, G.C. Produção e qualidade de frutos de laranjeira 'Pera' em função de fontes e doses de boro. Revista Brasileira de Fruticultura, Jaboticabal, v. 28, n.2, p. 328-330, 2006.

BROWN, P.H.; SHELP, B.J. Boron mobility in plants. Plant and Soil, Dordrecht, v.193, n.1-2, p.85-101, 1997.

FAO. Production-crops. Disponível em: $\leq \mathrm{http}: / /$ faostat.fao.org/site/339/default.aspx $>$. Acesso em: 12 de jan. 2014.

GALLI, J.A.; PALHARINI, M.C. de A.; FISCHER, I.H.; MECHELOTTO, M.D. Boro: efeito na produção e qualidade de frutos de diferentes variedades de manga. Pesquisa \& Tecnologia, Campinas, v. 9, n. 2, 2012.

GENÚ, P.J. de C.; PINTO, A.C. de A. A Cultura da mangueira. Brasília: Embrapa Informação Tecnológica, 2002. 454 p.

GOLDBERG, S.; FORSTER, H.S.; HEICK, E.L. Boron adsorption mechanisms on oxides, clay minerals, and soils inferred from ionic strength effects. Soil Science Society of America Journal, Madison, v.57, n.3, p.704-708, 1993.

IBGE. Banco de dados agregados: sistema IBGE de recuperação automática: SIDRA. Disponível em: <www.ibge.gov.br>. Acesso em: 18 jan. 2014.

IBRAF - Instituto Brasileiro de Frutas. Frutas frescas: exportação. 2014. Disponível em: <www. ibraf.org.br $>$. Acesso em: 10 jan. 2014. 
LEMISKA, A.; PAULETTI, V.; CUQUEL, F.L.; ZAWADNEAK, M.A.C. Produção e qualidade da fruta do morangueiro sob influência da aplicação de boro. Ciência Rural, Santa Maria, v.44, n.4, p.622628, 2014.

MOAZZAM, A.; TAHIR, F.M.; SHAHZAD, J.; MAHMOOD, N. Effect of foliar application of micronutrients on the quality of mango (Mangiferaindica L.) cv. Dashehari fruit. Mycopathologia, Dordrecht, v.9, n.1, p.25-28, 2011.

PERICA, S.; BELLALOUI, N.; GREVE, C.; HU, H.; BROWN, P.H. Boron transport and soluble carbohydrate concentrations in olive. Journal of the American Society Horticultural Science, Alexandria, v.126, p.291-296, 2001.

PLEGUEZUELO, C.R.R., ZUAZO, V.H.D.; FERNÁNDEZ, J.L.M.; TARIFA, D.F. Physicochemical quality parameters of mango (Mangifera indica $\mathrm{L}$.) fruits grown in a mediterranean subtropical climate (SE Spain). Journal of Agricultural Science and Technology, Tehran, v.14, n.2, p.365-374, 2012.

PRADO, R.M.; NATALE, W.; ROZANE, D.E. Níveis críticos de boro no solo e na planta para cultivo de mudas de maracujazeiro-amarelo1. Revista Brasileira de Fruticultura, Jaboticabal, v.28, n.2, p.305-309, 2006.

PROGRAMA BRASILEIRO PARA A MODERNIZAÇÃO DA HORTICULTURA. Normas de classificação de manga. São Paulo: Centro de Qualidade em Horticultura (CEAGESP), 2004. 6 p. (Documentos, 28).

QUAGGIO, J.A.; MATTOS JUNIOR, D.; CANTARELLA, H.; TANK JUNIOR, A. Fertilização com boro e zinco no solo em complementação à aplicação via foliar em laranjeira Pêra(1). Pesquisa Agropecuária Brasileira, Brasília, v.38, n.5, p.627634, 2003.
RAJPUT, C.B.S.; SINGH, B.P.; MISHRA, H. Effects of foliar application of boron on mango. Scientia Horticulturae, Amsterdam, v.5, n.4, p.311-313, 1976.

RAVEN, J.A. Short- and long distance transport of boric acid in plants. New Phytologist, London, v.84, p.231-249, 1980.

SÁ, A.A.; ERNANI, P.R.; NAVA, G.; AMARANTE, C.V.T.; PEREIRA, A.J. Influência de formas de aplicação de boro na qualidade e no rendimento de maçãs (Malus domestica). Revista Brasileira de Fruticultura, Jaboticabal, v.36, n.2, p.487- 494, 2014.

SARAN, P.L.; KUMAR, R. Boron deficiency disorders in mango (Mangifera indica): field screening, nutrient composition and amelioration by boron application. Indian Journal of Agricultural Sciences, New Delhi, v.81, n.6, p.506-510, 2011.

SILVA, A.C. da; SOUZA, A.P. de; LEONEL, S.;SOUZA, M.E. de; RAMOS, D.P.; TANAKA, A.A. Growth and flowering of five mango cultivar under subtropics conditions of Brazil. American Journal of Plant Sciences, Irvine, v.5, n.3, p.393-402, 2014.

SILVA, F.C. (Ed.). Manual de análises químicas de solos, plantas e fertilizantes. Brasília: Embrapa Informação Tecnológica, 2009. 627 p.

WÓJCIK, P.; WOJCIK, M.; KLAMKOWSKI, K. Response of apple trees to boron fertilization under conditions of low soil boron availability. Scientia Horticulturae, Amsterdam, v.116, p.58-64, 2008. 
CARACTERÍSTICAS FISICOQUÍMICAS DE LA GRASA DE SEMILLA DE VEINTE CULTIVARES DE MANGO (Mangifera indica L.) EN COLOMBIA ${ }^{1}$

\author{
MARTHA DEL PILAR LÓPEZ HERNÁNDEZ², ANGÉLICA PIEDAD SANDOVAL ALDANA², \\ JORGE ALBERTO VALENCIA MONTOYA ${ }^{3}$
}

RESUMEN - En la presente investigación se evaluó el rendimiento de extracción y propiedades fisicoquímicas de la grasa de semilla de 20 cultivares de mango (Mangifera Indica L.) en Colombia. Se emplearon semillas de frutos en estado maduro, procesadas en harina con el siguiente rango de composición proximal: grasa 6.73 \% - $11.8 \%$; proteína $4.3 \%$ - $6.9 \%$; fibra cruda $2.6 \%$ - $6.1 \%$ y cenizas $2.12 \%$ - 3.9 \%. Las características fisicoquímicas determinadas en el aceite crudo mostraron los siguientes rangos: índice de acidez $0.78 \%$ ácido oleico - 1.83 \% ácido oleico; índice de saponificación $121.77 \mathrm{mg} \mathrm{de} \mathrm{KOH/g} \mathrm{-} 233.88 \mathrm{mg} \mathrm{de} \mathrm{KOH/g;}$ índice de peróxidos $0.76 \mathrm{meq} \mathrm{O}_{2} / \mathrm{kg}-3.19 \mathrm{meq} \mathrm{O}_{2} / \mathrm{kg}$; índice de iodo $22.94 \mathrm{~g}$ de $\mathrm{I}_{2} / 100 \mathrm{~g}-32.52 \mathrm{~g} \mathrm{de} \mathrm{I}_{2} / 100$ g. El perfil de ácidos grasos presentó, en promedio, al ácido oleico (46.46\%) y el ácido esteárico (37.58 $\%)$ como componentes mayoritarios. La grasa obtenida no presentó un contenido considerable de fenoles totales. El estudio realizado demostró que la obtención de grasa comestible a partir de subproductos del mango, constituye una alternativa viable del aprovechamiento de recursos naturales pues los rendimientos en algunos cultivares fue alto y su composición química posibilita su aplicación en la sustitución de grasas vegetales o la posible generación de nuevos productos.

Palabras clave: Ácidos grasos, grasa, mango, Mangifera indica L., semilla, subproductos.

\title{
PHYSICOCHEMICAL CHARACTERISTICS OF SEED FAT FOR TWENTY MANGO (Mangifera indica L.) CULTIVARS IN COLOMBIA
}

\begin{abstract}
In the present investigation was evaluated yield extraction and some physicochemical properties of seed fat for 20 mango (Mangifera Indica L.) cultivars in Colombia. Seeds of mature fruit were processed in flour and were obtained the following composition: fat $6.73 \%-11.8 \%$, protein $4.3 \%-6.9 \%$, raw fiber $2.6 \%-6.1 \%$ and ash $2.12 \%-3.9 \%$. The physicochemical characteristics in the crude mango fat showed the following ranges: acid value $0.78 \%$ oleic acid $-1.83 \%$ oleic acid; saponification value 121.77 $\mathrm{mg} \mathrm{KOH} / \mathrm{g}-233.88 \mathrm{mg} \mathrm{KOH} / \mathrm{g}$; peroxide value $0.76 \mathrm{meq} \mathrm{O}_{2} / \mathrm{kg}-3.19 \mathrm{meq} \mathrm{O}_{2} / \mathrm{kg}$; iodine value $22.94 \mathrm{~g}$ $\mathrm{I}_{2} / 100 \mathrm{~g}-32.52 \mathrm{~g} \mathrm{I} / 100 \mathrm{~g}$. The fatty acid profile presented oleic acid $(46.46 \%)$ and stearic acid (37.58 $\%$ ) as the major components. The fat obtained showed no significant content of total phenols. The study showed that edible fat obtained from mango by-products is a viable alternative to enable full use of natural resources as some cultivars presented high yields with a chemical composition that define their application as vegetables fat substitution and also the possibility of new product generation
\end{abstract}

Index terms: By-products, fatty acids, fat, mango, Mangifera indica L., seed.

\footnotetext{
1(Trabalho 297-14). Recebido em:17-11-2014. Aceito para publicação em:08-04-2015.

${ }^{2}$ Ing. Agroindustrial, Joven Investigador, Corporación Colombiana de Investigación Agropecuaria (CORPOICA), Laboratorio de Ciencias Agroalimentarias. Kilómetro nueve vía Espinal-Ibagué, Tolima, Colombia. E-mail: mdlopez@ut.edu.co

${ }^{3} \mathrm{PhD}$. Ing. Química, Docente Investigador, Universidad del Tolima, Facultad de Ingeniería Agronómica, Ingeniería Agroindustrial, Calle 67 No. 53-108 B. Santa Helena, Ibagué -Tolima, Colombia. E-mail: apsandovala@ut.edu.co

${ }^{4}$ Ing. Agrónomo, Investigador Profesional, Corporación Colombiana de Investigación Agropecuaria (CORPOICA). E-mail: jvalencia@corpoica.org.co
} 